\title{
Nonperturbative estimate of the heavy quark momentum diffusion coefficient
}

\author{
A. Francis, ${ }^{1}$ O. Kaczmarek, ${ }^{2}$ M. Laine, ${ }^{3,4}$ T. Neuhaus, ${ }^{5, *}$ and H. Ohno ${ }^{6,7}$ \\ ${ }^{1}$ Department of Physics and Astronomy, York University, Toronto, Ontario M3J1P3, Canada \\ ${ }^{2}$ Faculty of Physics, University of Bielefeld, 33501 Bielefeld, Germany \\ ${ }^{3}$ ITP, AEC, University of Bern, Sidlerstrasse 5, 3012 Bern, Switzerland \\ ${ }^{4}$ Helsinki Institute of Physics, P.O. Box 64, 00014 University of Helsinki, Finland \\ ${ }^{5}$ Institute for Advanced Simulation, FZ Jülich, 52425 Jülich, Germany \\ ${ }^{6}$ Center for Computational Sciences, University of Tsukuba, Ibaraki 305-8577, Japan \\ ${ }^{7}$ Physics Department, Brookhaven National Laboratory, Upton, New York 11973, USA
}

(Received 24 August 2015; published 9 December 2015)

\begin{abstract}
We estimate the momentum diffusion coefficient of a heavy quark within a pure SU(3) plasma at a temperature of about $1.5 T_{\mathrm{c}}$. Large-scale Monte Carlo simulations on a series of lattices extending up to $192^{3} \times 48$ permit us to carry out a continuum extrapolation of the so-called color-electric imaginary-time correlator. The extrapolated correlator is analyzed with the help of theoretically motivated models for the corresponding spectral function. Evidence for a nonzero transport coefficient is found and, incorporating systematic uncertainties reflecting model assumptions, we obtain $\kappa=(1.8-3.4) T^{3}$. This implies that the "drag coefficient," characterizing the time scale at which heavy quarks adjust to hydrodynamic flow, is $\eta_{D}^{-1}=(1.8-3.4)\left(T_{\mathrm{c}} / T\right)^{2}(M / 1.5 \mathrm{GeV}) \mathrm{fm} / \mathrm{c}$, where $M$ is the heavy quark kinetic mass. The results apply to bottom and, with somewhat larger systematic uncertainties, to charm quarks.
\end{abstract}

DOI: 10.1103/PhysRevD.92.116003

PACS numbers: 11.10.Wx, 11.15.Ha, 12.38.Mh, 12.39.Hg

\section{INTRODUCTION}

Within linear response theory the rate at which a system relaxes towards local thermal equilibrium is characterized by quantities known as transport coefficients. Different transport coefficients parametrize different types of perturbations. If we focus on a conserved particle number, such as a quark flavor, ${ }^{1}$ which is initially distributed unevenly, such as in a broad jet cone, then the relevant transport coefficient is the diffusion coefficient. In QCD, there is a separate diffusion coefficient related to light flavors, and to heavy flavors such as charm and bottom. A closely related quantity is the electrical conductivity, which can be expressed as a weighted sum over the flavor diffusion coefficients.

The determination of transport coefficients related to strong interactions at temperatures of a few hundred $\mathrm{MeV}$ is an important goal for lattice QCD. It is a challenging task, given that lattice QCD is formulated in a Euclidean spacetime whereas transport coefficients are real-time quantities (for a review, see Ref. [1]). Nevertheless, motivated by the ongoing heavy ion collision program, large-scale efforts have been undertaken. For example, for the light-quark contribution to electrical conductivity, recent works can be found in Refs. [2-7].

The focus of the present study is the diffusion coefficient associated with heavy quarks. It has been one of the major

\footnotetext{
${ }^{*}$ Deceased.

${ }^{1}$ Weak interactions play no role within the lifetime $\lesssim 20 \mathrm{fm} / \mathrm{c}$ of a fireball generated in a heavy ion collision.
}

qualitative discoveries of the heavy ion collision program at RHIC and LHC that charm quarks appear to flow about as efficiently as light quarks do (see, e.g., Refs. [8-10] and references therein). That a flow develops is an example of a relaxation towards local thermal equilibrium. It is then a theoretical challenge to explain this behavior from the laws of QCD [11]. A next-to-leading order (NLO) computation in perturbation theory indicates the presence of a large correction towards strong interactions [12], and strong interactions have also been observed in $\mathcal{N}=4$ superYang-Mills theory [13-16]. Furthermore classical lattice gauge theory simulations [17] and analyses in the confined phase [18-22] are consistent with strong interactions, and various other approaches are being pursued in the same vein [23-29] (for a review, see Ref. [30]). Heavy quark diffusion also happens to pose an ideal ground for more general theoretical investigations of nonequilibrium thermodynamics [31]. In any case, ultimately the problem needs to be addressed with lattice simulations.

For $M \gg \pi T$, where $M$ is the heavy quark "kinetic mass," the lattice determination of the heavy quark diffusion coefficient can be reduced to a purely gluonic measurement [32]. Here we report the final results of a multiyear study of the relevant observable in the deconfined phase of pure $\mathrm{SU}(3)$ gauge theory. It has been demonstrated a while ago that, with advanced numerical methods, a signal can be obtained at a fixed lattice spacing [33-35]. However the issues of renormalization, taking the continuum limit, and analytic continuation had not been brought into conclusion. Even though further improvements are needed and can be 
foreseen on all of these fronts, the purpose of the current paper is to present an analysis which offers a "minimal" practical answer to the main open points.

The plan of this paper is the following. After introducing the observable and reviewing the techniques that have been used for its determination and the data sets that have been collected (Sec. II), we carry out a continuum extrapolation in Sec. III. The simple structure of the associated spectral function, as revealed by previous theoretical works, allows us to attack the difficult problem of analytic continuation through tightly constrained models, as well as a variant of the so-called Backus-Gilbert method (Sec. IV). Our final results and some future prospects are presented in Sec. V.

\section{MEASUREMENTS}

Using heavy quark effective theory, the force felt by a heavy quark as it propagates through a gluon plasma can be related to a "color-electric correlator" [32] (cf. also Ref. [15]),

$$
\begin{aligned}
G_{\mathrm{E}}(\tau) & \equiv-\frac{1}{3} \sum_{i=1}^{3} \frac{\left\langle\operatorname{Re} \operatorname{Tr}\left[U(\beta ; \tau) g E_{i}(\tau, \mathbf{0}) U(\tau ; 0) g E_{i}(0, \mathbf{0})\right]\right\rangle}{\langle\operatorname{Re} \operatorname{Tr}[U(\beta ; 0)]\rangle} \\
\beta & \equiv \frac{1}{T}
\end{aligned}
$$

where $g E_{i}$ denotes the color-electric field, $T$ the temperature, and $U\left(\tau_{2} ; \tau_{1}\right)$ a Wilson line in the Euclidean time direction. The discretization of this correlator is not unique; we employ the proposal of Ref. [32], illustrated in Fig. 1.

We have measured the discretized correlator in quenched lattice QCD, employing the standard Wilson gauge action, at a temperature corresponding to about $1.5 T_{\mathrm{c}}$ (the parameters of the simulations are shown in Table I). In order to obtain a signal for our observable, advanced statistical error reduction techniques are required. As has been discussed in more detail in Refs. [34,36], we have employed 1000 additional multilevel updates [37,38] for the electric field insertions, and link integration $[39,40]$ for the straight lines between them. Moreover, in order to reduce discretization effects, the imaginary-time separations are tree-level improved [34] in analogy with the procedure previously used in other contexts $[38,41]$.

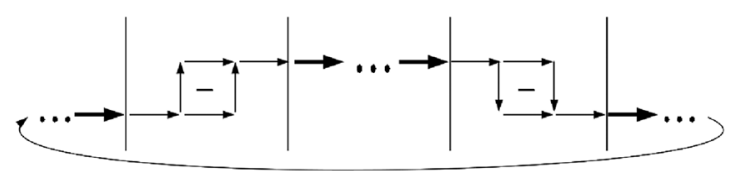

FIG. 1. One possible discretization of Eq. (2.1) [32]. Different techniques have been used for improving on the statistical signal originating from the links denoted with thick lines, and from the electric field insertions delineated with the vertical lines (cf. the text).
The resulting correlators are illustrated in Fig. 2 (left). For better visibility, the correlators have been normalized to (cf. Ref. [32])

$$
G_{\text {norm }}(\tau) \equiv \pi^{2} T^{4}\left[\frac{\cos ^{2}(\pi \tau T)}{\sin ^{4}(\pi \tau T)}+\frac{1}{3 \sin ^{2}(\pi \tau T)}\right],
$$

which diverges as $1 /\left(\pi^{2} \tau^{4}\right)$ at $\tau \ll \beta$. The correlators have also been multiplied by a renormalization factor as will be explained in Sec. III. As discussed in more detail in Ref. [34], volume dependence lies below statistical uncertainties in our measurements. In the following, we therefore consider a fixed spatial extent in units of the temperature, $L_{\mathrm{s}}=4 / T$.

\section{CONTINUUM EXTRAPOLATION}

Given the lattice data at tree-level improved distances, we carry out an extrapolation to the continuum limit. For this the lattice-measured correlator needs to be multiplied by a renormalization factor:

$$
G_{\mathrm{E}, \mathrm{cont}}(\tau) \equiv \mathcal{Z}_{\mathrm{E}} G_{\mathrm{E}, \mathrm{latt}}(\tau)
$$

A one-loop perturbative computation yields [45]

$$
\mathcal{Z}_{\mathrm{E}, \text { pert }}=1+0.079 \times \frac{6}{\beta_{0}}+\mathcal{O}\left(0.079 \times \frac{6}{\beta_{0}}\right)^{2}
$$

where $\beta_{0} \equiv 6 / g_{0}^{2}$ is the coupling of the plaquette term in the Wilson action (cf. Table I). Ultimately renormalization should be carried out nonperturbatively; however, the small coefficient of the one-loop term in Eq. (3.2) suggests that perturbative renormalization should yield a reasonable approximation.

Measured results at four or five different values of $N_{\tau}$ (depending on $\tau T$; cf. Fig. 2) are interpolated to the values of $\tau T$ that are shown in Table II. At fixed $\tau T$ we extrapolate the correlator in $a^{2}$ to the continuum limit. The procedure is illustrated in Fig. 2 (right) for selected values of $\tau T$. The resulting continuum limit is shown in Fig. 3 (left), and the results are tabulated in Table II. The results and errors of continuum-extrapolated correlation functions were obtained from a combined jackknife analysis. A covariance matrix for the continuum correlator was also estimated within this analysis (however the errors are strongly correlated even at large $\tau$ separations; the covariance matrix has very small eigenvalues and oscillatory eigenvectors, and therefore its inverse is of limited practical use in fitting).

\section{MODELING THE SPECTRAL FUNCTION}

Given the data for the imaginary-time correlator, the next task is to constrain the corresponding spectral function. The relation of a spectral function $\rho_{\mathrm{E}}(\omega)$ to the corresponding imaginary-time correlator $G_{\mathrm{E}}(\tau)$ reads 
TABLE I. The lattices included in the current analysis. Conversions to units of $t_{0}$ are based on Ref. [42] for the improved Wilson discretization ("imp"); on Refs. [42,43] for the clover discretization ("clov") of the observable defining $t_{0}$ [44]; and on Ref. [42] for $r_{0}$ [41]. Note however that the clover case is not well represented by our ansatz [42]: $\chi^{2} /$ d.o.f. $\approx 42$. Conversions to $T_{\mathrm{c}}$ are based on Ref. [42].

\begin{tabular}{lcccccccc}
\hline \hline$\beta_{0}$ & $N_{\mathrm{s}}^{3} \times N_{\tau}$ & Confs & $T \sqrt{t_{0}}$ (imp) & $T /\left.T_{\mathrm{c}}\right|_{t_{0}} ^{(\mathrm{imp})}$ & $T \sqrt{t_{0}}$ (clov) & $T /\left.T_{\mathrm{c}}\right|_{t_{0}} ^{\text {(clov) }}$ & $T r_{0}$ & $T /\left.T_{\mathrm{c}}\right|_{r_{0}}$ \\
\hline 6.872 & $64^{3} \times 16$ & 172 & 0.3770 & 1.52 & 0.3805 & 1.53 & 1.116 & 1.50 \\
7.035 & $80^{3} \times 20$ & 180 & 0.3693 & 1.48 & 0.3739 & 1.50 & 1.086 & 1.46 \\
7.192 & $96^{3} \times 24$ & 160 & 0.3728 & 1.50 & 0.3790 & 1.52 & 1.089 & 1.46 \\
7.544 & $144^{3} \times 36$ & 693 & 0.3791 & 1.52 & 0.3896 & 1.57 & 1.089 & 1.46 \\
7.793 & $192^{3} \times 48$ & 223 & 0.3816 & 1.53 & 0.3955 & 1.59 & 1.084 & 1.45 \\
\hline \hline
\end{tabular}

$$
G_{\mathrm{E}}(\tau)=\int_{0}^{\infty} \frac{\mathrm{d} \omega}{\pi} \rho_{\mathrm{E}}(\omega) \frac{\cosh \left[\omega\left(\frac{\beta}{2}-\tau\right)\right]}{\sinh \left[\frac{\omega \beta}{2}\right]}
$$

Even though an inversion of this relation is possible in principle [46] (after the subtraction of short-distance singularities [47]), the problem is ill posed in practice: large variations of $\rho_{\mathrm{E}}$ may lead to small changes of $G_{\mathrm{E}}$. Therefore, it is important to constrain the allowed form of $\rho_{\mathrm{E}}$ from general considerations. Here we do this by fixing the functional form of $\rho_{\mathrm{E}}$ at small $(\omega \ll T)$ and large frequencies $(\omega \gg T)$. Subsequently theoretically motivated interpolations between the two limits are proposed. We consider the spatial volume to be infinite and assume $\rho_{\mathrm{E}}$ to be a smooth function of $\omega$, as is generally the case in an interacting thermal system.

\section{A. IR and UV asymptotics}

In the infrared (IR) regime $(\omega \ll T)$, the heavy quark momentum diffusion coefficient can be defined as [32]

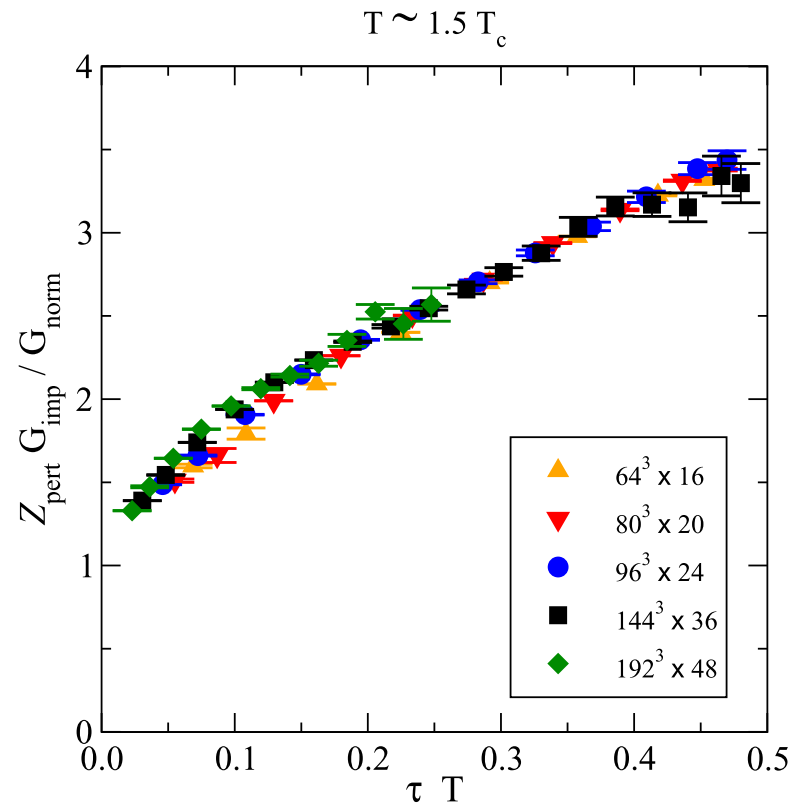

$$
\kappa \equiv \lim _{\omega \rightarrow 0} \frac{2 T \rho_{\mathrm{E}}(\omega)}{\omega} .
$$

The approach to this limit appears to be smooth: resummed perturbative computations [32] and numerical simulations within classical lattice gauge theory [17], as well as strongcoupling computations in analogous theories [15,48], suggest that $\rho_{\mathrm{E}}$ has no transport peak but is rather a monotonically increasing function. Therefore, we define the infrared asymptotics through the simplest form consistent with Eq. (4.2):

$$
\phi_{\mathrm{IR}}(\omega) \equiv \frac{\kappa \omega}{2 T} .
$$

Consider then the ultraviolet (UV) regime $(\omega \gg T)$. Thanks to asymptotic freedom, the UV behavior of the spectral function can be computed in perturbation theory. Denoting by $g^{2}$ the QCD gauge coupling renormalized in the $\overline{\mathrm{MS}}$ scheme, and $a_{s} \equiv \alpha_{s} / \pi \equiv g^{2} /\left(4 \pi^{2}\right)$, the result has the structure

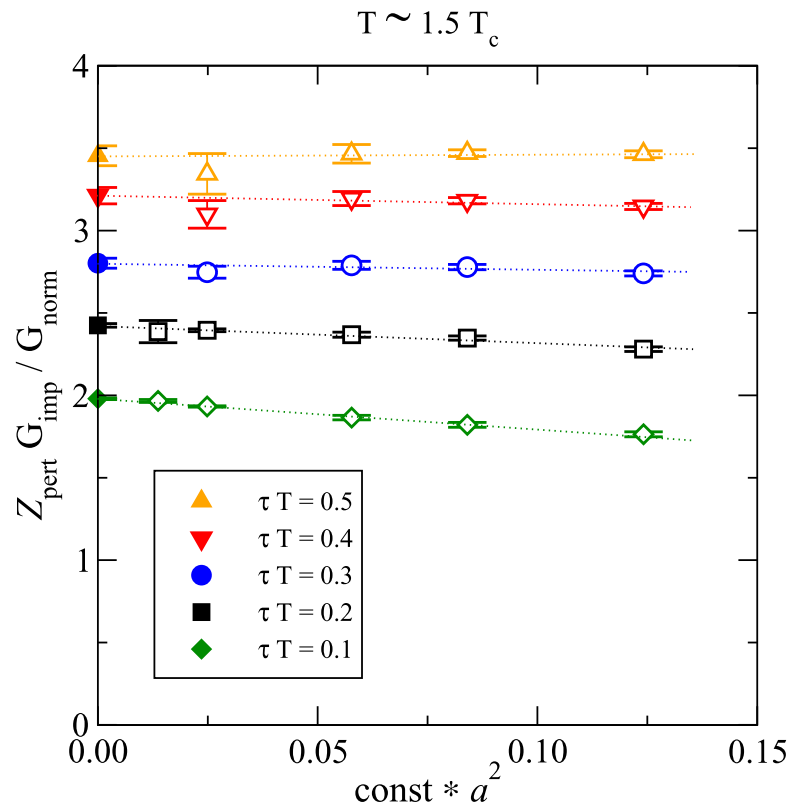

FIG. 2 (color online). Left: Lattice data after perturbative renormalization and the use of tree-level improved distances. Right: Illustrations of continuum extrapolations at selected values of $\tau T$ (our final errors are based on a jackknife analysis and differ somewhat from those shown here). 
TABLE II. The continuum-extrapolated correlator, normalized to Eq. (2.2). The errors are statistical and result from linear extrapolations in $1 / N_{\tau}^{2}$ to the continuum limit (cf. the text).

\begin{tabular}{lc}
\hline \hline$\tau T$ & $\frac{Z_{\text {pert }} G_{\text {latt }}}{G_{\text {norm }}}$ \\
\hline $4 / 48$ & $1.8727(43)$ \\
$5 / 48$ & $2.0044(31)$ \\
$6 / 48$ & $2.1157(25)$ \\
$7 / 48$ & $2.2133(31)$ \\
$8 / 48$ & $2.3001(44)$ \\
$9 / 48$ & $2.3793(56)$ \\
$10 / 48$ & $2.4548(65)$ \\
$11 / 48$ & $2.5300(72)$ \\
$12 / 48$ & $2.6070(79)$ \\
$13 / 48$ & $2.6865(86)$ \\
$14 / 48$ & $2.7686(92)$ \\
$15 / 48$ & $2.8529(96)$ \\
$16 / 48$ & $2.9391(98)$ \\
$17 / 48$ & $3.0260(101)$ \\
$18 / 48$ & $3.1124(107)$ \\
$19 / 48$ & $3.1962(117)$ \\
$20 / 48$ & $3.2745(130)$ \\
$21 / 48$ & $3.3441(145)$ \\
$22 / 48$ & $3.4005(161)$ \\
$23 / 48$ & $3.4390(176)$ \\
$24 / 48$ & $3.4538(190)$ \\
\hline \hline
\end{tabular}

$$
\rho_{\mathrm{E}}(\omega) \stackrel{\omega \gg T}{=}\left[\rho_{\mathrm{E}}(\omega)\right]_{T=0}+\mathcal{O}\left(\frac{g^{4} T^{4}}{\omega}\right) .
$$

The leading thermal correction is consistent with the pattern expected from the operator product expansion [49]

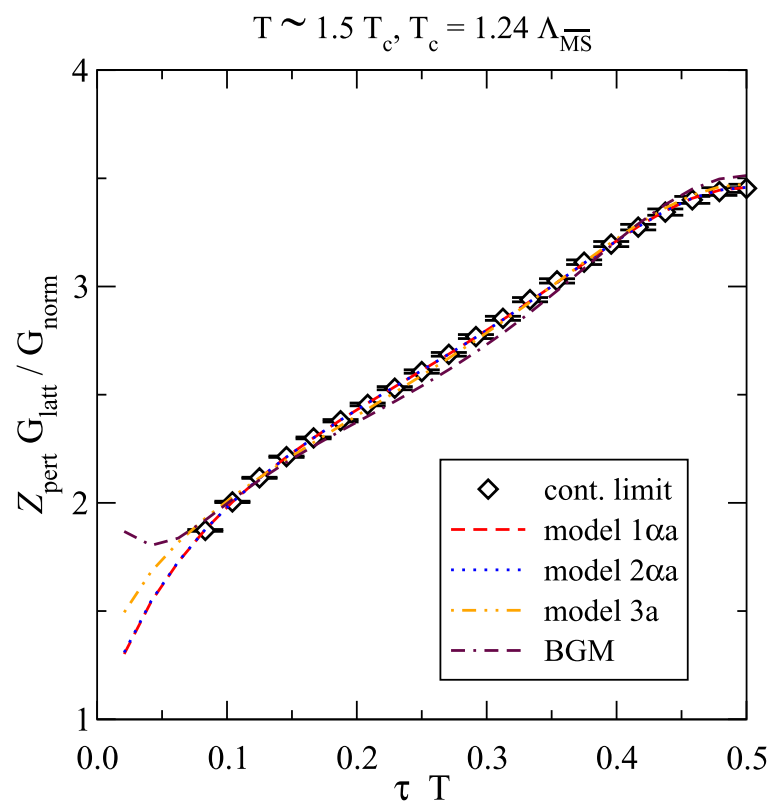

(for $N_{\mathrm{f}}=0$ the coefficient of this correction is negative [50]). The vacuum part has the form

$$
\begin{aligned}
{\left[\rho_{\mathrm{E}}(\omega)\right]_{T=0}=} & \frac{g^{2} C_{\mathrm{F}} \omega^{3}}{6 \pi}\left[r_{10}+\left(r_{20}+r_{21} \ell\right) a_{s}\right. \\
& \left.+\left(r_{30}+r_{31} \ell+r_{32} \ell^{2}\right) a_{s}^{2}+\mathcal{O}\left(a_{s}^{3}\right)\right]
\end{aligned}
$$

where $C_{\mathrm{F}} \equiv\left(N_{\mathrm{c}}^{2}-1\right) /\left(2 N_{\mathrm{c}}\right)=4 / 3$ and $\ell \equiv \ln \left(\bar{\mu}^{2} / \omega^{2}\right)$, with $\bar{\mu}$ denoting the renormalization scale. The three first coefficients read [50]

$r_{10}=1$

$r_{20}=N_{\mathrm{c}}\left(\frac{149}{36}-\frac{11 \ln 2}{6}-\frac{2 \pi^{2}}{3}\right)-N_{\mathrm{f}}\left(\frac{5}{9}-\frac{\ln 2}{3}\right)$,

$r_{21}=\frac{11 N_{\mathrm{c}}-2 N_{\mathrm{f}}}{12}$

where $N_{\mathrm{f}}$ denotes the number of light dynamical quarks $\left(N_{\mathrm{f}}=0\right.$ in our study). The coefficient $r_{30}$ and higher-order terms are presently unknown. However, the general structure of Eq. (4.5), together with the knowledge that $\rho_{\mathrm{E}}$ requires no renormalization in dimensional regularization [32], is sufficient for determining the asymptotics of $\rho_{\mathrm{E}}$.

Indeed, suppose that we choose the renormalization scale as $\bar{\mu}=\omega$ for $\omega \gg \Lambda_{\overline{\mathrm{MS}}}$. Then $\ell=0, a_{s} \sim \ln ^{-1}\left(\omega / \Lambda_{\overline{\mathrm{MS}}}\right)$, and we get

$$
\left[\rho_{\mathrm{E}}(\omega)\right]_{T=0} \stackrel{\omega \gg T}{=} \phi_{\mathrm{UV}}^{(a)}(\omega)\left[1+\mathcal{O}\left(\frac{1}{\ln \left(\omega / \Lambda_{\overline{\mathrm{MS}}}\right)}\right)\right],
$$

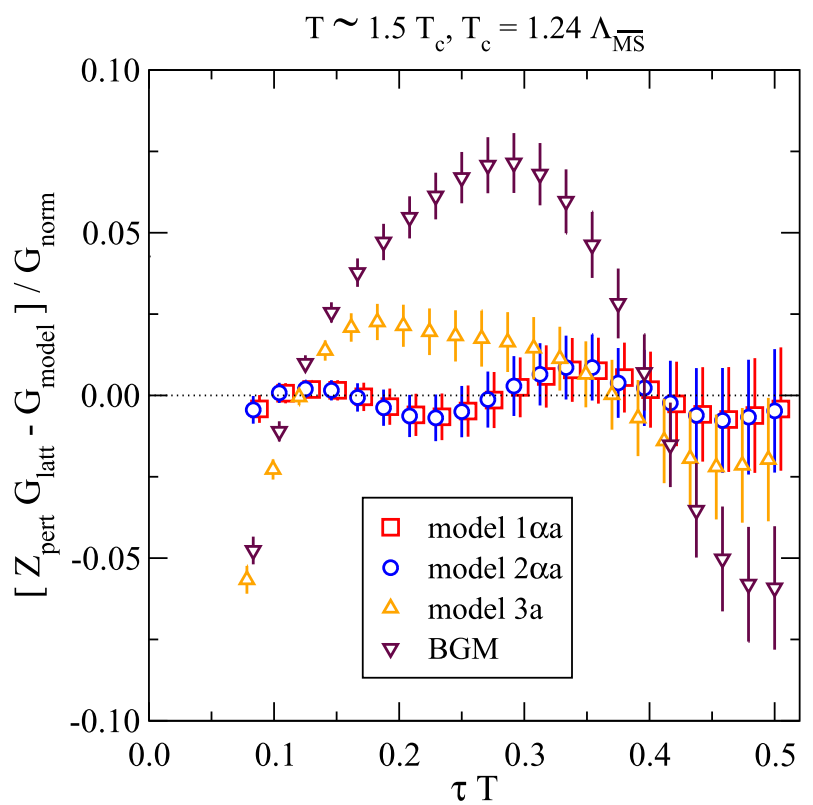

FIG. 3 (color online). Left: Continuum-extrapolated lattice data (cf. Table II) and examples of model results, as described in the text. The results have been normalized to Eq. (2.2). Right: The differences between the data and the models. For better visibility, the data points corresponding to models $1 \alpha a$ and $3 a$ have been displaced slightly. 
where we have defined

$$
\phi_{\mathrm{UV}}^{(a)}(\omega) \equiv \frac{g^{2}\left(\bar{\mu}_{\omega}\right) C_{\mathrm{F}} \omega^{3}}{6 \pi}, \quad \bar{\mu}_{\omega} \equiv \max (\omega, \pi T) .
$$

Formally, we can reduce the correction to be of quadratic rather than linear order in $\ln ^{-1}\left(\omega / \Lambda_{\overline{\mathrm{MS}}}\right)$ by including $r_{20}$ in the asymptotics, so let

$$
\phi_{\mathrm{UV}}^{(b)}(\omega) \equiv \phi_{\mathrm{UV}}^{(a)}(\omega)\left[1+\left(r_{20}+r_{21} \ell\right) a_{s}\left(\bar{\mu}_{\omega}\right)\right] .
$$

However, since the convergence of an expansion proceeding in inverse logarithms could be slow, and since our knowledge of $\omega / \Lambda_{\overline{\mathrm{MS}}}=(\omega / T) \times\left(T / \Lambda_{\overline{\mathrm{MS}}}\right)$ is imperfect due to uncertainties in $T / \Lambda_{\overline{\mathrm{MS}}}$ [42], we treat Eqs. (4.10) and (4.11) on an equal footing in the following. For evaluating $g^{2}\left(\bar{\mu}_{\omega}\right)$ and $a_{s}\left(\bar{\mu}_{\omega}\right)$, we have used four-loop running [51].

\section{B. Interpolations}

Equations (4.3) and (4.10) determine the limiting behaviors of the spectral function. For $\omega \ll T$, given the flatness observed in nonperturbative simulations [17], we expect corrections to $\phi_{\mathrm{IR}}$ to be given by a convergent power series in $\omega$. For $\omega \gg T$ the corrections are suppressed by inverse logarithms of $\omega / T$, and it is important to account for these corrections. In order to incorporate the different types of corrections in the two regimes, we map the interval $\omega \in(0, \infty)$ to the interval $(0,1)$ by introducing

$x \equiv \ln \left(1+\frac{\omega}{\pi T}\right) \in(0, \infty), \quad y \equiv \frac{x}{1+x} \in(0,1)$.

For small $y$ we have

$$
y \approx x \approx \frac{\omega}{\pi T}, \quad \omega \ll T,
$$

whereas around the other end we get

$$
1-y \approx \frac{1}{x} \approx \frac{1}{\ln [\omega /(\pi T)]}, \quad \omega \gg T .
$$

So, we can capture corrections to the asymptotics by a function in $y$ which vanishes at $y=0$ and $y=1$ and is a polynomial or a power series in between. A convenient choice is to employ trigonometric functions:

$$
e_{n}^{(\alpha)}(y) \equiv \sin (\pi n y)
$$

Another possibility, in principle preferable if we know that corrections are only quadratic in the variables in Eqs. (4.13) and (4.14) [cf. the discussion preceding Eq. (4.11)], is

$$
e_{n}^{(\beta)}(y) \equiv \sin (\pi y) \sin (\pi n y) .
$$

With these bases we are led to define general models $(\mu \in\{\alpha, \beta\}, i \in\{a, b\})$ :

$$
\begin{aligned}
\operatorname{model} 1: \rho_{\mathrm{E}}^{(1 \mu i)}(\omega) \equiv & {\left[1+\sum_{n=1}^{n_{\max }} c_{n} e_{n}^{(\mu)}(y)\right] } \\
& \times\left[\phi_{\mathrm{IR}}(\omega)+\phi_{\mathrm{UV}}^{(i)}(\omega)\right],
\end{aligned}
$$

$\operatorname{model} 2: \rho_{\mathrm{E}}^{(2 \mu i)}(\omega) \equiv\left[1+\sum_{n=1}^{n_{\max }} c_{n} e_{n}^{(\mu)}(y)\right]$

$$
\times \sqrt{\left[\phi_{\mathrm{IR}}(\omega)\right]^{2}+\left[\phi_{\mathrm{UV}}^{(i)}(\omega)\right]^{2}} .
$$

The difference between these interpolations is that the latter imposes a more rapid crossover from the IR to the UV asymptotics; for instance, for large $\omega$ we have $\sqrt{\phi_{\mathrm{IR}}^{2}+\phi_{\mathrm{Uv}}^{2}} \approx$ $\phi_{\mathrm{UV}}+\frac{\phi_{\mathrm{R}}^{2}}{2 \phi_{\mathrm{UV}}} \sim \omega^{3}+T^{4} / \omega$ in qualitative accordance with the functional form expected from Eq. (4.4). ${ }^{2}$ Finally, we also consider a simple two-parameter ansatz separating the IR and UV regimes completely:

$$
\operatorname{model} 3: \rho_{\mathrm{E}}^{(3 i)}(\omega) \equiv \max \left[\phi_{\mathrm{IR}}(\omega), c \phi_{\mathrm{UV}}^{(i)}(\omega)\right]
$$

where $c$ is treated as a free parameter, reflecting uncertainties in the renormalization factor in Eq. (3.2) [in practice we find $c=1.05(1)$ from the fit for $i=a]$.

\section{Fitting strategy}

The inversion of Eq. (4.1) represents an ill-posed problem, so it should not come as a surprise that it is, in general, not possible to find a "stable" fit describing the data. In other words, the $\chi^{2}$ function may possess an extremely shallow minimum, or multiple minima. Empirically we find, however, that these ambiguities are largely related to the UV behavior of the spectral function. With some further input, the UV behavior can be stabilized, yet the IR coefficient $\kappa$ in which we are mostly interested remains fairly unaffected.

Concretely, we have implemented two strategies for the fitting, which incorporate an implicit or explicit stabilization of the UV contribution. After defining ${ }^{3}$

$$
\chi^{2} \equiv \sum_{\tau_{i}}\left[\frac{G_{\text {meas }}\left(\tau_{i}\right)-G_{\text {model }}\left(\tau_{i}\right)}{\delta G_{\text {meas }}\left(\tau_{i}\right)}\right]^{2},
$$

\footnotetext{
${ }^{2}$ Note that, for $n_{\max } \rightarrow \infty$, such a construction can be viewed as a general parametrization of an arbitrary spectral function. We call our parametrizations "models" because the problems discussed in Sec. IV C necessitate keeping $n_{\max }$ relatively small and stabilizing the subsequent fits through additional input.

${ }^{3}$ We have also carried out tests with the covariance matrix, but in its full form this does not yield sensible results as alluded to above. If a sufficient infrared cutoff is imposed on the smallest eigenvalues, the results are consistent within errors with the procedure described here.
} 
where $\delta G_{\text {meas }}$ denote the errors from Table II, the following alternatives are considered:

(i) In the first strategy, we search for a minimum of $\chi^{2}$ for a result based on Eq. (4.1), taking a jackknife sample as the measured result. The search is performed using the algorithm described in Ref. [52], taking $\kappa / T^{3}=1.0, c_{n}=0.0$ as a starting point. The search is stopped after 200 iterations, by which time an excellent representation of the data has in general been reached (with $\chi^{2} /$ d.o.f. $\sim 0.3-0.5$ for a typical sample from the jackknife ensemble), with coefficients $\left|c_{n}\right| \lesssim 0.2$.

(ii) In the second strategy, we stabilize the fit by imposing the constraint

$$
\rho_{\mathrm{E}}^{(n \mu i)}\left(\omega_{\max }\right) \equiv \phi_{\mathrm{UV}}^{(i)}\left(\omega_{\max }\right),
$$

where we choose $\omega_{\max } \equiv 1000 T$. This constraint imposes a relation between $\kappa$ and $c_{n}$, so that there are only $n_{\max }$ free parameters. We find that with this constraint a unique minimum of $\chi^{2}$ can always be found, with $\chi^{2} /$ d.o.f. $\sim 0.2-0.6$. The practical search was performed with the routine described in Ref. [53]. Because of the artificial constraint at large $\omega / T$, the fit is carried out only to distances $\tau T \geq 6 / 48$.

In the following we describe results from both procedures, demonstrating that they yield similar results for $\kappa$, particularly for our preferred "model 2" [cf. Eq. (4.18)].

In the case of the two-parameter model 3 defined in Eq. (4.19)), a global minimum of $\chi^{2}$ is readily found without further input. The price to pay for this stability is that the model does not describe the data particularly well, having $\chi^{2} /$ d.o.f. $\approx 17.5$ for $\phi_{\mathrm{UV}}^{(a)}$ (we do not show results for $\phi_{\mathrm{Uv}}^{(b)}$ for which $\chi^{2} /$ d.o.f. $\left.\approx 68\right)$.

In addition to these fits, we have also made use of a variant of the Backus-Gilbert method (BGM) [54,55] (see Refs. [56,57] for previous applications in lattice QCD). The goal of this method is not to reconstruct the spectral function itself but rather an averaged version thereof. With very precise data, the averaging kernel could be made optimally narrow in a certain sense. In practice, the finite precision of the data necessitates a regularization of an ill-defined matrix inversion; this is characterized by a parameter $\lambda$ for which we use $\lambda=10^{-4}$ rather than the theoretically optimal $\lambda=1$ (cf. Ref. [57]). As a consequence, our estimate of the infrared limit of the spectral function amounts to a weighted average over the range $0 \leq \omega \lesssim 10 T$. Fortunately, if there is little structure in this range, the result should be reasonable. Moreover it is possible to rescale the kernel in order to further remove known structures; we insert $\rho_{\mathrm{E}}(\omega)=\left[\rho_{\mathrm{E}}(\omega) / \phi(\omega)\right] \phi(\omega)$ in Eq. (4.1) with $\phi(\omega) \equiv(\omega \beta)^{3} / \tanh ^{2}(\omega \beta / 4)$ and subsequently include $\phi$ in the kernel. To keep the matrix size manageable, only $\tau / \beta=(4-21) / 48$ were used for this analysis. If the final result for the averaged $\rho_{\mathrm{E}}(\omega)$ is inserted back into Eq. (4.1), it yields a representation of the original data with $\chi^{2} /$ d.o.f. $\approx 41$.

We end by remarking that, apart from these novel approaches, we have also applied the standard maximum entropy method (MEM) to our problem. MEM requires the specification of a default model as input. If we take as the default model our model 1 or 2, which yield a Euclidean correlator agreeing with lattice data everywhere (within statistical errors), then MEM reproduces the default model as its output (within statistical errors). In other words, MEM does not help to constrain the result beyond our analysis.

\section{Estimation of $\kappa$}

We now turn to estimating $\kappa$. In Sec. IV B we have introduced a large class of fit functions: eight separate models $\rho_{\mathrm{E}}^{(n \mu i)}, n \in\{1,2\}, \mu \in\{\alpha, \beta\}, i \in\{a, b\}$, each of which depends on a parameter $n_{\max }$. In addition we have introduced two fitting strategies (cf. Sec. IV C). In the following, we choose $n_{\max }=4,5$ and demonstrate that, for a given model, both strategies yield similar results within error bars. (We have also used $n_{\max }=3$ and $n_{\max }=6$; these yield nothing qualitatively new.) The agreement is more significant for our preferred model 2. The spread between the models is interpreted as an indication of the systematic uncertainty of our determination. Model $3 a$ and the BGM approach are included as further crosschecks; in the BGM case the spread of results originating

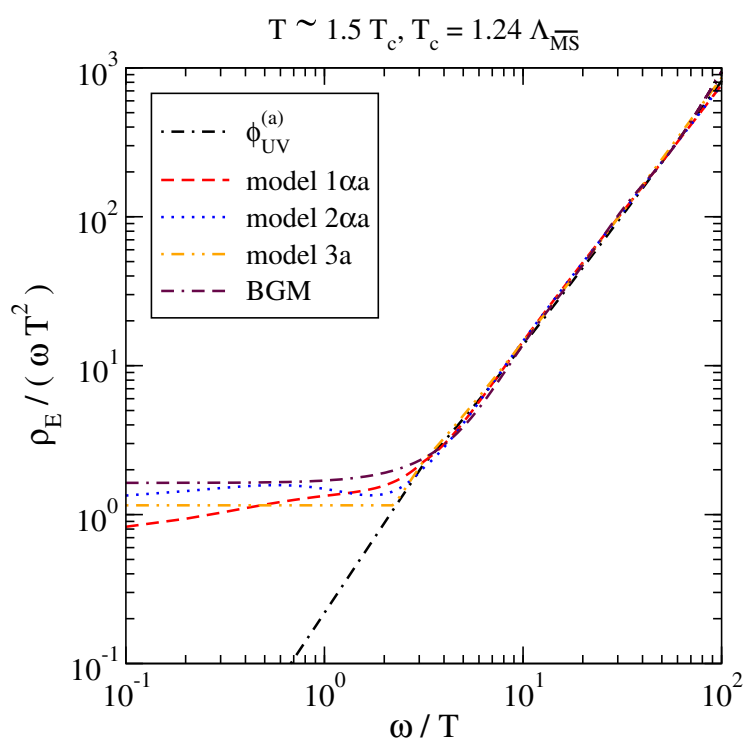

FIG. 4 (color online). Examples of model spectral functions, compared with $\phi_{\mathrm{UV}}^{(a)}$ from Eq. (4.10). In the BGM case the curve shown has been obtained by replacing the full covariance matrix with a diagonal one possessing the errors shown in Table II, but we have obtained BGM results with full and IR-regularized covariance matrices as well; they are included in the average shown in Fig. 5. 


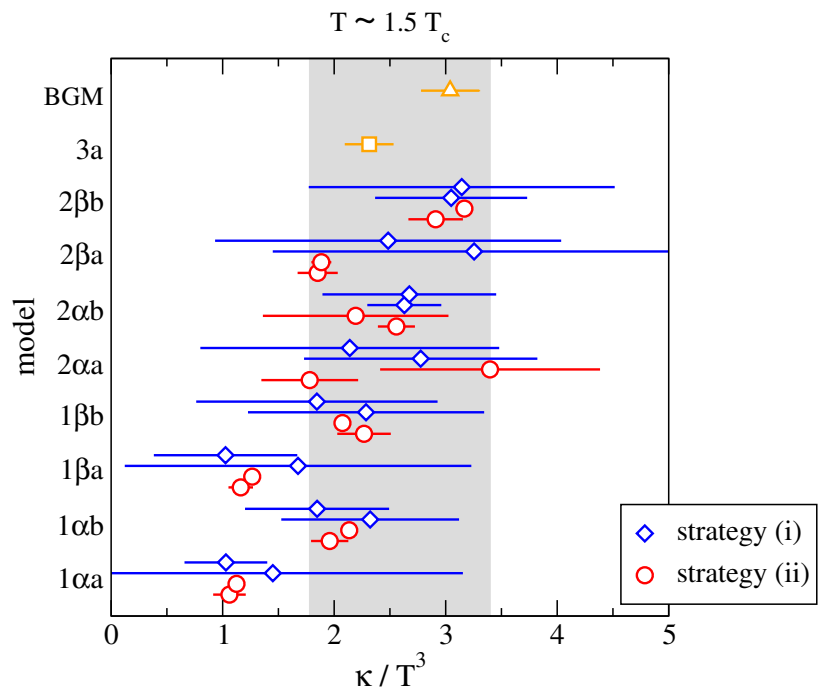

FIG. 5 (color online). Fit results based on different models (cf. Sec. IV B) and different fitting strategies (cf. Sec. IV C). In each case, the lower data point corresponds to $n_{\max }=4$, the higher to $n_{\max }=5$. For model $3 a$ [cf. Eq. [24]] and BGM (cf. Sec. IV C) systematic errors are larger than those shown. The gray band illustrates our final estimate, given in Eq. (4.22) and based on models 2, $3 a$ and BGM.

from different versions of covariance matrices (cf. caption of Fig. 4) is included in the error shown.

The fits are carried out to samples from a jackknife ensemble, and the ensemble is used for determining statistical errors. Results from the different models are illustrated in Figs. 3-5 [the fits in Figs. 3 and 4 are based on strategy (i)]. Given that model 2 yields more stable results within the other variations, and that it is theoretically better justified than model 1 [cf. the discussion below Eq. (4.18))], we make use of it in the following. Based on the central values from Fig. 5 for models 2, $3 a$ and BGM we estimate

$$
\kappa / T^{3}=1.8 \ldots 3.4
$$

This result is illustrated in Fig. 5 with a gray band. Given that the result is dominated by systematic uncertainties which may be asymmetric but are essentially impossible to estimate, we refrain from citing a central value here. ${ }^{4}$

\section{CONCLUSIONS}

The purpose of this paper has been to estimate the heavy quark momentum diffusion coefficient, defined through Eqs. (2.1), (4.1) and (4.2). Compared with previous works [33-35], we have carried out a continuum extrapolation of the imaginary-time correlator (cf. Sec. III) and discussed

\footnotetext{
${ }^{4}$ It is remarkable that our result is consistent with previous ones obtained at a single lattice spacing and with very rough modeling of the spectral shape $[34,35]$. However, the uncertainties related to the continuum extrapolation and to analytic continuation are now much closer to being under control.
}

the systematics related to estimating the corresponding spectral function (cf. Sec. IV). The final result of our analysis is given in Eq. (4.22). It is remarkable that, despite the ill-posed nature of analytic continuation, our novel approach permits us to obtain relatively stable results and to strongly constrain the order of magnitude of the heavy quark momentum diffusion coefficient in the continuum limit.

In the nonrelativistic limit (i.e. for a heavy quark mass $M \gg \pi T) \kappa$ is related to the diffusion coefficient $D$ as $D=2 T^{2} / \kappa$, and to the drag coefficient as $\eta_{D}=\kappa /(2 M T)$, where $M$ is a heavy quark kinetic mass [32]. The drag coefficient can also be interpreted as the kinetic equilibration time scale associated with heavy quarks: $\tau_{\text {kin }}=\eta_{D}^{-1}$. For a conversion to physical units, we use $r_{0} T_{\mathrm{c}}=$ $0.7457(45)$ [42] and $r_{0}=0.47(1)$ fm [58]. With these conversions, our result $\kappa / T^{3}=1.8-3.4$ yields an estimate for the time scale associated with the kinetic equilibration of heavy quarks,

$\tau_{\text {kin }}=\frac{1}{\eta_{D}}=(1.8 \ldots 3.4)\left(\frac{T_{\mathrm{c}}}{T}\right)^{2}\left(\frac{M}{1.5 \mathrm{GeV}}\right) \mathrm{fm} / \mathrm{c}$.

Close to $T_{\mathrm{c}}$, charm quark kinetic equilibration appears therefore to be almost as fast as that of light partons, for which a time scale $\sim 1 \mathrm{fm} / \mathrm{c}$ is generally considered appropriate. For the diffusion coefficient we obtain

$$
D T=0.59 \ldots 1.1 .
$$

This can be compared with the values $D T \gtrsim 0.13$ obtained for light quarks in quenched QCD in the continuum limit $[4,5]$. It would be "natural" for the $D$ of heavy quarks to be of the same order of magnitude but somewhat larger than that of light quarks, given that heavy quarks should feel slightly weaker interactions.

It is also interesting to compare our result for $\kappa$ with an NLO perturbative computation [12]. That result is of the form $\kappa / T^{3}=\alpha_{\mathrm{s}}^{2}\left(c_{1}+c_{2} \alpha_{\mathrm{s}}^{1 / 2}\right)$, where $c_{1}, c_{2}$ are coefficients given in Ref. [12] ( $c_{1}$ involves a logarithmic dependence on $\alpha_{\mathrm{s}}$ ). In the absence of corrections of relative order $\alpha_{\mathrm{s}}$, it is not possible to estimate the renormalization scale at which $\alpha_{\mathrm{s}}$ should be evaluated. Nevertheless, the result shown in Fig. 3 of Ref. [12] agrees with our Eq. (4.22) if we set $\alpha_{\mathrm{s}}=0.20-0.26$, which is in full accordance with the range generally used in heavy ion collision phenomenology.

Many possible directions can be envisaged for future investigations. Improved statistical precision is crucial for moving towards model-independent analytic continuation [47]. Other temperatures than just $T \sim 1.5 T_{\mathrm{c}}$ should be considered. The determination of the renormalization factor $\mathcal{Z}_{\mathrm{E}}$ in Eq. (3.1) should be promoted to the nonperturbative level. It would be important to understand whether the heavy-mass limit is justified for charm quarks (or only for 
bottom quarks); this can in principle be studied by using the full relativistic formulation for measuring current-current correlation functions $[59,60]$, even though then the structure of the spectral function is more complicated and analytic continuation is even more difficult to get under reasonable control. Finally, estimating effects from dynamical quarks is important for phenomenological applications.

\section{ACKNOWLEDGMENTS}

We thank J. Langelage and M. Müller for collaboration at initial stages of this project and H. Sandmeyer for his work on the $80^{3} \times 20$ ensemble. M. L. is grateful to H. B. Meyer for helpful discussions. Our work has been supported in part by the DFG under Grant No. GRK881, by the SNF under Grant No. 200020-155935, by the European Union through HadronPhysics3 (Grant No. 283286) and ITN STRONGnet (Grant No. 238353), and by the Väisälä Foundation. Simulations were performed using JARA-HPC resources at the RWTH Aachen and JSC Jülich (projects JARA0039 and JARA0108), JUDGE/JUROPA at the JSC Jülich, the OCuLUS Cluster at the Paderborn Center for Parallel Computing, and the Bielefeld GPU cluster.
[1] H. B. Meyer, Transport Properties of the Quark-Gluon Plasma: A lattice QCD perspective, Eur. Phys. J. A 47, 86 (2011).

[2] S. Gupta, The electrical conductivity and soft photon emissivity of the QCD plasma, Phys. Lett. B 597, 57 (2004).

[3] G. Aarts, C. Allton, J. Foley, S. Hands, and S. Kim, Spectral Functions at Small Energies and the Electrical Conductivity in Hot, Quenched Lattice QCD, Phys. Rev. Lett. 99, 022002 (2007).

[4] H.-T. Ding, A. Francis, O. Kaczmarek, F. Karsch, E. Laermann, and W. Soeldner, Thermal dilepton rate and electrical conductivity: An analysis of vector current correlation functions in quenched lattice QCD, Phys. Rev. D 83, 034504 (2011).

[5] Y. Burnier and M. Laine, Towards flavour diffusion coefficient and electrical conductivity without ultraviolet contamination, Eur. Phys. J. C 72, 1902 (2012).

[6] B. B. Brandt, A. Francis, H. B. Meyer, and H. Wittig, Thermal correlators in the $\rho$ channel of two-flavor QCD, J. High Energy Phys. 03 (2013) 100.

[7] G. Aarts, C. Allton, A. Amato, P. Giudice, S. Hands, and J. I. Skullerud, Electrical conductivity and charge diffusion in thermal QCD from the lattice, J. High Energy Phys. 02 (2015) 186.

[8] B. Abelev et al. (ALICE Collaboration), D Meson Elliptic Flow in Noncentral $\mathrm{Pb}-\mathrm{Pb}$ Collisions at $\sqrt{s_{\mathrm{NN}}}=2.76 \mathrm{TeV}$, Phys. Rev. Lett. 111, 102301 (2013).

[9] A. Adare et al. (PHENIX Collaboration), Heavy-quark production and elliptic flow in $\mathrm{Au}+\mathrm{Au}$ collisions at $\sqrt{s_{N N}}=62.4 \mathrm{GeV}$, Phys. Rev. C 91, 044907 (2015).

[10] R. Vertesi (STAR Collaboration), Heavy Flavor Measurements at STAR, arXiv:1410.3959.

[11] G. D. Moore and D. Teaney, How much do heavy quarks thermalize in a heavy ion collision?, Phys. Rev. C 71, 064904 (2005).

[12] S. Caron-Huot and G. D. Moore, Heavy Quark Diffusion in Perturbative QCD at Next-to-Leading Order, Phys. Rev. Lett. 100, 052301 (2008).

[13] C. P. Herzog, A. Karch, P. Kovtun, C. Kozcaz, and L. G. Yaffe, Energy loss of a heavy quark moving through $\mathcal{N}=4$ supersymmetric Yang-Mills plasma, J. High Energy Phys. 07 (2006) 013.
[14] S. S. Gubser, Drag force in AdS/CFT, Phys. Rev. D 74, 126005 (2006).

[15] J. Casalderrey-Solana and D. Teaney, Heavy quark diffusion in strongly coupled $\mathcal{N}=4$ Yang Mills, Phys. Rev. D 74, 085012 (2006).

[16] S. Caron-Huot and G. D. Moore, Heavy quark diffusion in QCD and $\mathcal{N}=4$ SYM at next-to-leading order, J. High Energy Phys. 02 (2008) 081.

[17] M. Laine, G. D. Moore, O. Philipsen, and M. Tassler, Heavy Quark Thermalization in Classical Lattice Gauge Theory: Lessons for Strongly-Coupled QCD, J. High Energy Phys. 05 (2009) 014.

[18] M. Laine, Heavy flavour kinetic equilibration in the confined phase, J. High Energy Phys. 04 (2011) 124.

[19] M. He, R. J. Fries, and R. Rapp, Thermal Relaxation of Charm in Hadronic Matter, Phys. Lett. B 701, 445 (2011).

[20] L. M. Abreu, D. Cabrera, F. J. Llanes-Estrada, and J. M. Torres-Rincon, Charm diffusion in a pion gas implementing unitarity, chiral and heavy quark symmetries, Ann. Phys. (Amsterdam) 326, 2737 (2011).

[21] S. K. Das, S. Ghosh, S. Sarkar, and J. Alam, Heavy flavor suppression: Role of hadronic matter, Phys. Rev. D 88, 017501 (2013).

[22] V. Ozvenchuk, J. M. Torres-Rincon, P. B. Gossiaux, L. Tolos, and J. Aichelin, D-meson propagation in hadronic matter and consequences for heavy-flavor observables in ultrarelativistic heavy-ion collisions, Phys. Rev. C 90, 054909 (2014).

[23] H. van Hees, M. Mannarelli, V. Greco, and R. Rapp, Nonperturbative Heavy-Quark Diffusion in the QuarkGluon Plasma, Phys. Rev. Lett. 100, 192301 (2008).

[24] F. Riek and R. Rapp, Quarkonia and Heavy-Quark Relaxation Times in the Quark-Gluon Plasma, Phys. Rev. C 82, 035201 (2010).

[25] A. Beraudo, A. De Pace, M. Monteno, M. Nardi, and F. Prino, Heavy flavors in heavy-ion collisions: Quenching, flow and correlations, Eur. Phys. J. C 75, 121 (2015).

[26] T. Song, H. Berrehrah, D. Cabrera, J. M. Torres-Rincon, L. Tolos, W. Cassing, and E. Bratkovskaya, Tomography of the Quark-Gluon-Plasma by Charm Quarks, Phys. Rev. C 92, 014910 (2015). 
[27] S. Cao, G. Y. Qin, and S. A. Bass, Energy loss, hadronization and hadronic interactions of heavy flavors in relativistic heavy-ion collisions, Phys. Rev. C 92, 024907 (2015).

[28] R. Abir and A. Majumder, Drag induced radiative loss from semi-hard heavy quarks, arXiv:1506.08648.

[29] K. Saraswat, P. Shukla, and V. Singh, Constraining heavy quark energy loss using $B$ and $D$ meson measurements in heavy ion collision at RHIC and LHC energies, Nucl. Phys. A943, 83 (2015).

[30] A. Beraudo, Dynamics of heavy flavor quarks in high energy nuclear collisions, Nucl. Phys. A931, 145 (2014).

[31] Y. Akamatsu, Langevin dynamics and decoherence of heavy quarks at high temperatures, Phys. Rev. C 92, 044911 (2015).

[32] S. Caron-Huot, M. Laine, and G. D. Moore, A way to estimate the heavy quark thermalization rate from the lattice, J. High Energy Phys. 04 (2009) 053.

[33] H. B. Meyer, The errant life of a heavy quark in the quarkgluon plasma, New J. Phys. 13, 035008 (2011).

[34] A. Francis, O. Kaczmarek, M. Laine, and J. Langelage, Towards a non-perturbative measurement of the heavy quark momentum diffusion coefficient, Proc. Sci., LATTICE2011 (2011) 202 [arXiv:1109.3941].

[35] D. Banerjee, S. Datta, R. Gavai, and P. Majumdar, Heavy Quark Momentum Diffusion Coefficient from Lattice QCD, Phys. Rev. D 85, 014510 (2012).

[36] A. Francis, O. Kaczmarek, M. Laine, M. Müller, T. Neuhaus, and H. Ohno, Towards the continuum limit in transport coefficient computations, Proc. Sci., LATTICE2013 (2014) 453 [arXiv:1311.3759].

[37] M. Lüscher and P. Weisz, Locality and exponential error reduction in numerical lattice gauge theory, J. High Energy Phys. 09 (2001) 010.

[38] H. B. Meyer, A calculation of the shear viscosity in SU(3) gluodynamics, Phys. Rev. D 76, 101701 (2007).

[39] G. Parisi, R. Petronzio, and F. Rapuano, A Measurement of the String Tension Near the Continuum Limit, Phys. Lett. B 128, 418 (1983).

[40] P. de Forcrand and C. Roiesnel, Refined methods for measuring large distance correlations, Phys. Lett. B 151, 77 (1985).

[41] R. Sommer, A new way to set the energy scale in lattice gauge theories and its applications to the static force and $\alpha_{\mathrm{S}}$ in SU(2) Yang-Mills theory, Nucl. Phys. B411, 839 (1994).

[42] A. Francis, O. Kaczmarek, M. Laine, T. Neuhaus, and H. Ohno, Critical point and scale setting in SU(3) plasma: An update, Phys. Rev. D 91, 096002 (2015).

[43] M. Asakawa, T. Hatsuda, T. Iritani, E. Itou, M. Kitazawa, and H. Suzuki, Accurate Determination of Reference Scales for Wilson Gauge Action from Yang-Mills Gradient Flow, arXiv:1503.06516.

[44] M. Lüscher, Properties and uses of the Wilson flow in lattice QCD, J. High Energy Phys. 08 (2010) 071; 03 (2014) 92(E).

[45] C. Christensen and M. Laine (unpublished).

[46] G. Cuniberti, E. De Micheli, and G. A. Viano, Reconstructing the thermal Green functions at real times from those at imaginary times, Commun. Math. Phys. 216, 59 (2001).

[47] Y. Burnier, M. Laine, and L. Mether, A test on analytic continuation of thermal imaginary-time data, Eur. Phys. J. C 71, 1619 (2011).

[48] S. S. Gubser, Momentum fluctuations of heavy quarks in the gauge-string duality, Nucl. Phys. B790, 175 (2008).

[49] S. Caron-Huot, Asymptotics of thermal spectral functions, Phys. Rev. D 79, 125009 (2009).

[50] Y. Burnier, M. Laine, J. Langelage, and L. Mether, Colourelectric spectral function at next-to-leading order, J. High Energy Phys. 08 (2010) 094.

[51] T. van Ritbergen, J. A. M. Vermaseren, and S. A. Larin, The four-loop $\beta$-function in quantum chromodynamics, Phys. Lett. B 400, 379 (1997).

[52] S. Singer and S. Singer, Efficient implementation of the Nelder-Mead Search Algorithm, Applied Numerical Analysis \& Computational Mathematics 1, 524 (2004) (available as the NAG routine E04CBF).

[53] F. James and M. Roos, Minuit: A System for Function Minimization and Analysis of the Parameter Errors and Correlations, Comput. Phys. Commun. 10, 343 (1975).

[54] G. Backus and F. Gilbert, The Resolving Power of Gross Earth data, Geophys. J. Int. 16, 169 (1968).

[55] W. H. Press, S. A. Teukolsky, W. T. Vetterling, and B. P. Flannery, Numerical Recipes: The Art of Scientific Computing (Cambridge University Press, Cambridge, England, 2007).

[56] H. B. Meyer, Calculation of the Bulk Viscosity in SU(3) Gluodynamics, Phys. Rev. Lett. 100, 162001 (2008).

[57] B. B. Brandt, A. Francis, H. B. Meyer, and D. Robaina, The pion quasiparticle in the low-temperature phase of QCD, Phys. Rev. D 92, 094510 (2015).

[58] R. Sommer, Scale setting in lattice QCD, Proc. Sci., LATTICE2013 (2014) 015 [arXiv:1401.3270].

[59] H. T. Ding, A. Francis, O. Kaczmarek, F. Karsch, H. Satz, and W. Soeldner, Charmonium properties in hot quenched lattice QCD, Phys. Rev. D 86, 014509 (2012).

[60] H. Ohno, Quarkonium correlation functions at finite temperature in the charm to bottom region, Proc. Sci., LATTICE2013 (2013) 172 [arXiv:1311.4565]. 